\section{Kinetics of Photosynthesis and Allied Processes}

THE photosynthetic process in the living plant consists of two separate reactions, namely, the primary photosynthetic reaction which has no temperature coefficient, and the dark or Blackman reaction which restores the conditions disturbed by the primary reaction and has a temperature coefficient. The velocity of the primary reaction is proportional to the concentration of the chlorophyll on the irradiated surface, the intensity of light and the concentration of the hydrated carbon dioxide. If these factors be denoted by $a, I$ and $P$, respectively, the initial velocity will be expressed by $k_{1} a I P$, and the velocity at time $t$ will be $k_{1} I P(a-x)$. The dark reaction is known to be unimolecular, and its velocity at time $t$ will be $k_{2} x e^{-Q / R T}$. It is obvious that a photostationary state will be established when the velocities of the primary and Blackman reactions become equal, and if this state be established at time $t$, then the observed rate of photosynthesis will be given by

$$
y=k_{1} I P(a-x)=k_{2} x e^{-Q / R T}
$$

Dividing by $k_{1} I P x$ we have

$$
\frac{y}{k_{1} I P x}=\frac{a-x}{x}=\frac{k_{2}}{k_{1} I P} e^{-Q / R T}
$$

But $k_{1} I P x=k_{1} I P a-y$, where $k_{1} I P a$ is the initial rate of photosynthesis, and for any one set of conditions is a constant, and if this be denoted by $K$ we have :

$$
\log \frac{y}{K-y}=\log \frac{k_{2}}{k_{1} I P}-\frac{Q^{1}}{T^{\prime}}
$$

where $Q^{1}=Q / 2 \cdot 303 \mathrm{R}$.

This formula expresses with great accuracy Emerson's four series of observations of the variation with temperature in the rate of photosynthesis with Chlorella ${ }^{1}$. The value of $K$, as indicated by the formula, decreases with the chlorophyll concentration, and the four values of $Q$ are $30,105,29,309$, $29,959,29,905$ calories, respectively, with a mean value of 29,819 calories.

The formula also explains the fact, first discovered by Blackman and more recently observed by Warburg $^{2}$, that the temperature coefficient becomes unity when the intensity of light is small.

By the elimination of $x$ from (1) we obtain

$$
y=\frac{k_{1} I P a k_{2} e^{-Q / R T}}{k_{1} I P+k_{2} e^{-Q / R T}}
$$

from which may be derived

$\frac{I}{y}=\frac{1}{k_{1} a P}+\frac{I}{a k_{2} e^{-Q / R T}}$ and $\frac{P}{y}=\frac{1}{k_{1} a I}+\frac{P}{a k_{2} e^{-Q / R T}}$

These equations indicate linear relations between $I / y$ and $I$, and between $P / y$ and $P$, when the temperature is constant. Warburg's observations ${ }^{2}$ of the relations between the rate of photosynthesis and the intensity of light and the hydrated $\mathrm{CO}_{2}$ concentration, respectively, at constant temperature are expressed by the above linear equations.

Let the case be postulated of heterogeneous catalysis in which the primary catalytic reaction is effected by the de-activation of the catalyst, and the de-activated catalyst is re-activated by a thermal reaction. If $a$ be the initial concentration of the active catalyst, $c$ the concentration of the catalyte, and $d$ the concentration of the substance which re-activates the catalyst, then, if $c$ and $d$ be large and sensibly constant, equation (2) becomes

$$
\log \frac{y}{K-y}=\log \frac{k_{2} d}{k_{1} c}-\frac{Q^{1}}{T},
$$

where $K=k_{1} a c$ and $y$ is the observed rate of catalysis. Equation (3) now becomes

$$
y=\frac{k_{1} a c k_{2} d e^{-Q / R T}}{k_{1} a c+k_{2} d e^{-Q / R T}}
$$

whence we have

$$
\frac{c}{y}=\frac{1}{k_{1} a}+\frac{c}{k_{2} d e^{-Q / R T}}
$$

which indicates a linear relation between $c / y$ and $c$ when $d$ and $T$ are constant. Under these conditions, equation (5) may be written in the form

$$
y=\frac{k^{1} a c}{c+K_{m}}
$$

which is the well-known Michaelis equation for enzyme action when $a$ and $c$ are the concentrations of enzyme and substrate, respectively. The wide applicability of the Michaelis equation and the close analogy between it and the equations expressing the rate of photosynthesis suggest that enzyme action is also a cyclic process, in which the primary catalytic reaction is effected by the de-activation of the enzyme, and the de-activated enzyme is re-activated in a thermal reaction, possibly by the co-enzyme.

E. C. C. BaLY.

L. B. Morgan

\section{The University,} Liverpool.

1 J. Gen. Physiol., 12, 623 ; 1929.

'Biochem. Z., 100, 258 ; 1919.

\section{Formation of Vortex Rings from a Liquid Drop}

A DROP of liquid heavier than water, for example blue or red ink, submerged quietly into still water, encounters the resistance of the medium and becomes flatter in its form in the course of its descending motion. The central part of the disc thus formed soon disappears, and consequently a ring form results.

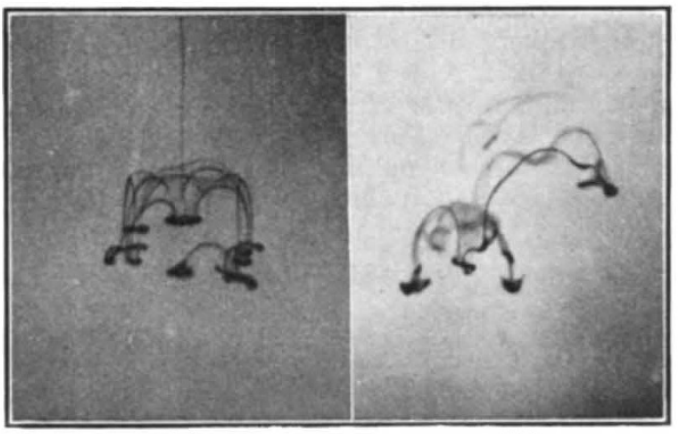

(a)

(b)

FTa. 1.

This is a very slowly whirling vortex ring. As it proceeds, the radius of the ring is augmented, and it begins to disintegrate into several minor vortex rings. Each of the secondary vortex rings proceeds in the same manner as the primary one, and disintegrates as well. The process of disintegration may be continued so far as the material remains ample. 